\title{
Estudio de pertinencia de la oferta educativa de docentes en educación básica, en la provincia de El Oro, Ecuador
}

\section{Study relevance of provision of basic education teachers in the province of El Oro, Ecuador}

\author{
Enrique Espinoza Freire
}

\author{
Universidad Técnica de Machala, P.O. BOX 462 Machala - El ORO. Ecuador
}

DOI: https://doi.org/10.33017/RevECIPeru2015.0014/

\section{Resumen}

Estudio exploratorio y descriptivo, cuyo objetivo es determinar la pertinencia técnica y social de la carrera de formación de docentes en educación básica, de la Universidad Técnica de Machala, de ecuador, lo cual hace referencia a la capacidad de la institución educativa, para expresar respuestas coherentes e integrales a las necesidades y exigencias de la sociedad. En este sentido y para efectos del estudio se empleó metodología de corte teórico como: análisis y síntesis, para la revisión bibliográfica, así como el constructo teórico, además del análisis de resultados obtenidos producto de la aplicación de métodos empíricos como la encuesta, la revisión documental. También en el presente trabajo se analiza algunos indicadores de calidad educativa en referencia a la formación de maestros, así como el impacto en los servicios educativos por parte de los egresados y la percepción de los directivos educativos sobre las competencias docentes, así mismo se determinó en prospectiva el ingreso estudiantil a primer año de educación básica de 8.439, por año hasta el 2017; por otro lado, se obtuvo como resultado la demanda laboral que está en 4.456 plazas para los egresados de la carrera motivo del presente estudio. Además se revela la evaluación del programa para medir el cumplimiento de las políticas educativas por el sistema y el control de los parámetros de calidad establecidos en el Ecuador, con el fin de que el programa y los estudiantes beneficiados alcancen las competencias requeridas, para desempeñar los puestos de trabajo propios del perfil de salida diseñado y se cumpla con el desafío de formar un recurso humano más calificado para la labor formativa.

\section{Palabras clave: pertinencia social, formación de maestros, educación básica}

\begin{abstract}
Exploratory and descriptive study aimed at determining the technical and social relevance of the career training of teachers in basic education, the Technical University of Machala, in Ecuador, which refers to the ability of the school to express answers coherent and comprehensive to the needs and demands of society. In this sense and for purposes of the study methodology was used as a theoretical tool: analysis and synthesis, for the literature review and the theoretical construct, plus analysis of results due to the application of empirical methods as the survey, review documentary. Also in this paper some indicators of educational quality in reference to the training of teachers, and the impact on education services by graduates and perception of educational managers on teaching skills discussed, likewise determined in Prospective student entering first year of basic education of 8,439 per year until 2017; on the other hand, it resulted in labor demand is at 4,456 places for graduates of the career of this study reason. Further evaluation of the program is revealed to measure compliance with education policies by the system and control of the quality parameters established in Ecuador, so that the program benefited students and reach the appropriate skills to perform the work stations own output profile designed and meets the challenge of forming a more qualified human resources and the training work.
\end{abstract}

Keywords: social relevance, teacher training, basic education 


\section{Introducción}

Los estudios de pertinencia social cobran cada vez más un alto valor para establecer las estrategias de desarrollo tanto a nivel de toda la sociedad como en su expresión social, incluyendo sus manifestaciones por sectores de la producción y los servicios.

El criterio de pertinencia social se refiere a aquello (proceso $u$ objeto de que se trate) que se corresponde $y$ por tanto resulta adecuado $y$ oportuno, se basa por tanto en la subjetividad de los implicados. De tal suerte, constituye un referente valorativo de las acciones humanas que favorecen a todos. Por ello, todo lo que se significa como discordancia se asume como impertinente, o sea, que no corresponde con los intereses de los destinatarios.

Por tal razón, en estado de impertinencia del proceso de que se trate las decisiones que tomen los directivos, resultan ineficientes, ineficaces y finalmente, desviadas de los intereses y expectativas sociales de los interesados. De ahí el valor de la determinación de la pertinencia social de los procesos que se modelan, pues de sus resultados se logrará redimensionar el curso de dichos organismos.

La pertinencia social gira en torno a la economía pues se relaciona con el consumo humano. Supone una mayor racionalidad en el manejo de los recursos, ya sean financieros, materiales e incluso los recursos energéticos de los sistemas; para ello es vital elevar el grado de conciencia de los implicados sobre las posibilidades de reutilización y reciclaje de materiales y productos.

En el caso de los servicios educacionales, se entiende que la pertinencia (técnica y social) de los programas docentes está dada por el escenario futuro que se prevé, que impone a las instituciones especializadas de las que se trate, ofrecer servicios educativos de óptima calidad, contextualizados y orientados hacia las necesidades de los usuarios internos y externos de esos servicios.

El enfoque de pertinencia social es de tal valor que "exige que toda universidad pública sitúe sus funciones sustantivas en la complejidad y dinamismo del proceso de globalización a fin de reafirmar, con visión prospectiva, su papel científico en la sociedad y el compromiso de coadyuvar al desarrollo de las comunidades locales." [1]
Para la determinación de la pertinencia social se toma en consideración los recursos que se gastan, la cultura en que se brinda y la percepción de los que trabajan con los programas y de los que lo reciben.

Es pertinente a este trabajo la referencia a la Guía para la elaboración de los estudios de pertinencia social, factibilidad y estado del arte para la creación, modificación o liquidación de programas educativos, además se asume que pertinencia social "es la coherencia que existe entre el objetivo del programa educativo y el perfil de egreso establecidos en el plan de estudios, con las necesidades prevalecientes en el ámbito de influencia de la Universidad Técnica de Machala, con el mercado de trabajo o con proyectos de desarrollo local, regional, nacional e internacional." [2]

El trabajo de formación de maestros para la educación básica tiene en su centro el enfoque del Buen Vivir, que De acuerdo al objetivo 4 del Plan Nacional del Buen Vivir, que expresa: "Fortalecer las capacidades y potencialidades de la ciudadanía" (PNBV, 2013 - 2017). Por eso la Carrera de Licenciatura en Ciencias de Educación Básica propone formar profesionales comprometidos con la sociedad y el ambiente, a través del aprendizaje, la investigación, innovación tecnológica, la interculturalidad, la multi, trans e interdisciplinariedad del conocimiento y de los saberes, para vincularse con la sociedad bajo la perspectiva del bien público social aportando a la democratización del conocimiento para la garantía de derechos y la consecución de las siguientes metas:[3]

Aumentar al 95\%el porcentaje de personas entre 16 y 24 años con educación básica completa, al 78\%el porcentaje escolar de personas entre 18 y 24 años en bachillerato completo; Reducir al 3\%el abandono escolar en octavo grado en educación general básica y primer año de bachillerato;

Aumentar al 90\%el acceso a internet en establecimientos educativos, al 50\%la matrícula en educación superior, al 25\%la matrícula en Institutos Técnicos y Tecnológicos;

Alcanzar el $80 \%$ de titulación en educación superior y el $85 \%$ de profesores universitarios con títulos de cuarto nivel. (PNBV, 2013 - 2017).

El buen vivir es parte esencial de la estrategia de desarrollo de Ecuador en los últimos años. Se concibe como la forma de vida que permite el 
bienestar y la permanencia de la diversidad cultural y ambiental, es la armonía, igualdad, equidad y solidaridad; de allí que las metas descritas se consolidan en las tensiones y problemas de la Zona 7 que son las siguientes: a) universalización de la educación y diálogo de saberes, b) mejorar la calidad educativa, c) disminuir el analfabetismo entre indígenas y montubios, d) incremento del número de docentes en zonas rurales, e) incremento de la escolaridad a 10 años.

Con el propósito de ofrecer alternativas de solución al objetivo identificado del PNVB, a los conflictos y tensiones de la Zona 7, la Carrera de Licenciatura en Ciencias de Educación Básica aportará con saberes, a los sujetos que aprenden, a través del objetivo general "formar profesionales en Ciencias de la Educación Básica, comprometidos con la sociedad y el ambiente, con dominios epistemológicos en el paradigma de la complejidad, en modelos de Pedagogía contemporánea, plataformas virtuales, que promuevan a través de proyectos la vinculación con la colectividad, utilizando prácticas interculturales, generación de conocimientos para la investigación acción participativa y formativa que permita auscultar y conocer las necesidades $y$ demandas de profesionales así como la investigación generativa mediante emprendizajes colaborativos que conlleven a la solución de las tensiones con predominio de la Nuevas Tecnologías de la Información y Comunicación (NITCs) y de las Nuevas Tecnologías del Aprendizaje y la Comunicación al aula (NTACs) y del idioma extranjero.

El propósito expuesto se conseguirá con los objetivos especificados en pertinencia, ciudadanía intercultural, conocimientos, aprendizajes que contribuirán:

Aplicar metodologías desde un enfoque epistemológico, mediante la organización del conocimiento y aprendizaje respetando y promoviendo nuestra identidad cultural;

Fomentar la responsabilidad social, institucional y ambiental, con prácticas inclusivas que permita el desarrollo de una cultura de paz en el marco del Buen Vivir; Desarrollar en el estudiante competencias investigativas a través de contextos de generación de conocimientos y saberes educativos, tecnológicos y culturales capaces de impulsar convenios a nivel local e internacional;

Brindar las condiciones, medios e instrumentos necesarios para que los futuros Licenciados en
Ciencias de Educación Básica sean innovadores y generen emprendizajes con dominio de idiomas extranjeros de manera colaborativa y contextualizada, para la consecución de becas y pasantías. [4]

\section{Metodología}

El presente trabajo es de carácter descriptivo, exploratorio, por lo que se concibe como un proceso de indagación sobre el valor de una institución, programa o acción con fines educativos, éste es un proceso que parte del examen sistemático del fenómeno en sí, de su naturaleza, cualidades y relaciones entre los elementos que lo integran y con el sistema a que pertenecen para comprobar e interpretar sus logros, examinar planes de acción futuros, validar y hacer explícitos determinados valores.

En cuanto a la formación de maestros se reconocen como áreas prioritarias la búsqueda y utilización de metodologías pedagógicas innovadoras; la utilización de los servicios para las prácticas profesionales y también como medio para fundamentar la relación entre la tecnología educativa y la de servicios; la articulación del aprendizaje con el trabajo de los servicios educativos. En cada área se determinan los indicadores que permiten identificar la pertinencia del programa de estudio.

La metodología tiene un ordenamiento lógico y transcurre dentro del marco general del enfoque de sistemas. La secuencia incluye pasos o actividades imprescindibles donde cada momento de su realización tiene relación directa o indirecta, con el resto y en conjunto su observación garantiza la calidad del proceso. De ahí que fue de suma importancia considerar los siguientes aspectos: delimitar el objeto de estudio, los objetivos y propósitos; Precisar las variables, indicadores, así como la base y los criterios de comparación, realizar un serio proceso de recolección de la información que se precisa; también analizar e interpretar la información, sobre la base de los datos obtenidos, luego identificar los logros y deficiencias y valorar la calidad del programa.

\section{Procedimiento}

Con respecto de la recolección de información sobre la pertinencia técnica y social del programa educativo, fue necesario considerar el contexto educativo, del cual se tomó: los estudiantes, los profesores, los egresados, el currículo, la 
administración, el entorno institucional, el impacto social y la evaluación. [5]

En tal sentido, pero sin dejar de observar los procedimientos que garantizan la objetividad, confiabilidad, validez y fiabilidad para disminuir los sesgos provenientes del investigador, de los informantes o de los instrumentos de recolección, como principios que sustentan la calidad de los procesos educativos estudiados. [6]

De ahí que fue necesario para la recolección de información la utilización de la técnica de la encuesta y como instrumento el cuestionario, aplicado a egresados y directivos institucionales; el mismo que estaba estructurado para auscultar sobre la pertinencia del programa de estudios de la carrera. Además se utilizó el cuestionario entrevista con la finalidad de obtener el número de alumnos por paralelo y grados, profesores por área del conocimiento y egresados de la carrera que laboran en los diferentes instituciones educativas de la provincia de El Oro.

Por otro lado, el cuestionario también fue utilizado para determinar el total de instituciones educativas de la provincia [7], así como para la marginación de las instituciones por niveles de formación y especialidades. De este mismo modo, permitió la recogida de información referente a la tasa de natalidad y mortalidad desde el año 2010, así como, la proyección de la población en prospectiva para el año 2017. [8]

Otro de los recursos empleados fue el análisis documental que nos sirvió para rastrear e inventariar los documentos existentes y disponibles sobre la carrera de Educación Básica, como: número de egresados, diseño curricular, investigación, vinculación con la colectividad, proceso de evaluación, metodología, entre otros.

Para el análisis e interpretación de los resultados se empleó el método de análisis y síntesis, así como para la revisión y triangulación bibliográfica.

Así mismo, es importante resaltar que se tomó en cuenta las formalidades éticas para la investigación, como son: el respeto al encuestado, la confidencialidad de la información y el anonimato.

\section{Resultados y discusión}

Como parte del estudio de pertinencia se consideró el análisis estadístico de los centros educativos por nivel de Educación en la provincia de El Oro, documento extraído de la plataforma del Ministerio de Educación en el que consta además de las instituciones educativas el número de docentes y alumnos por género, su totalidad institucional y provincial, estos datos son muy importantes ya que la provincia de El Oro, es el área de influencia en lo que respecta a la cobertura de profesionales graduados en la Universidad Técnica de Machala.

Así tenemos que el total de centros educativos es de 917: de los cuales 19 ofertan educación inicial; 202 ofertan educación inicial y básica; 19 centros educativos tienen educación inicial, educación básica y bachillerato; los centros educativos que ofertan solo educación básica le corresponden 556; por otro lado tenemos que 125 centros educativos ofertan educación básica y bachillerato; además 1 centro educativo oferta bachillerato; 3 instituciones educativos tienen alfabetización y un centro educativo oferta educación artística, lo que nos deja claro que de la totalidad de instituciones educativas de la provincia de El Oro, 906 instituciones educativas ofertan educación básica materia de estudio en el presente análisis.

Tabla 1: Instituciones educativas por nivel de escolaridad de la provincia de El oro. Fuente: Ministerio de Educación del Ecuador 2013, compilado por autor

\begin{tabular}{|c|c|c|c|c|c|c|c|c|c|}
\hline 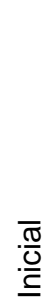 & 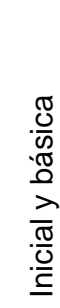 & 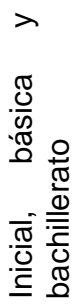 & 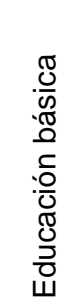 & 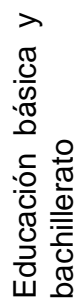 & 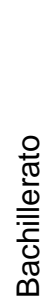 & 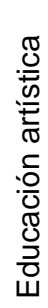 & 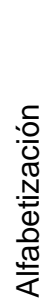 & 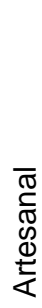 & 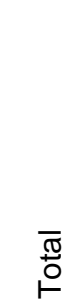 \\
\hline 9 & 202 & 19 & 556 & 125 & 1 & 1 & 3 & 1 & 917 \\
\hline
\end{tabular}

Con la información procesada por el Ministerio de Educación y facilitada a favor de la carrera de Educación Básica de la Universidad Técnica de Machala, se procedió a realizar un auscultamiento de manera aleatoria simple a 111 centros educativos de los diferentes cantones de la provincia, que se constituyen en el $12.25 \%$ del total de que ofertan educación básica, con el fin de cotejar datos sobre población estudiantil, pero sobre todo precisar la población escolar que se encuentra cursando la educación básica, la misma que fluctúa entre los 5 a 15 y hasta los 16 años de edad. 
Tabla 2: Población estudiantil de los 111 centros educativos analizados.

\begin{tabular}{|c|c|c|c|c|c|}
\hline$\frac{\tau}{\frac{\sigma}{\underline{J}}}$ & $\frac{N}{\frac{N}{O N}}$ & 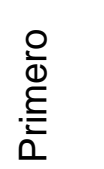 & $\begin{array}{l}\text { 응 } \\
\text { ᄃ } \\
\text { D } \\
\text { ஸ્ }\end{array}$ & $\begin{array}{l}\frac{0}{0} \\
\frac{0}{0} \\
-\end{array}$ & 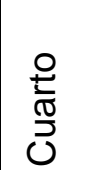 \\
\hline 1422 & 1221 & 2489 & 3395 & 3290 & 3386 \\
\hline $\begin{array}{l}\stackrel{0}{\frac{1}{5}} \\
\frac{1}{3}\end{array}$ & 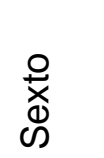 & 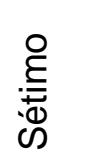 & $\begin{array}{l}\text { 坖 } \\
\text { 苟 }\end{array}$ & $\begin{array}{l}\text { O } \\
\text { Dे } \\
\text { ¿ }\end{array}$ & 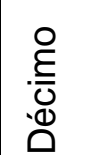 \\
\hline 3175 & 3117 & 2686 & 2398 & 2737 & 2719 \\
\hline total & \multicolumn{5}{|c|}{32035} \\
\hline
\end{tabular}

Como puede observarse, hay una cantidad relativamente estable de estudiantes potenciales para el ingreso a la escuela, con carácter perspectivo, lo cual garantiza una matrícula sostenida para el trabajo de los maestros.

Otro de los indicadores que se consideró en el estudio de pertinencia fue la opinión a 193 egresados de la carrera, dado sus características de formación y su relación con la realidad contextual en el que se desarrollan laboralmente, información relevante que nos permitirá fortalecer el diseño curricular.

Las preguntas realizadas están en el orden general como: nivel de satisfacción de la formación recibida, cumplimiento del perfil profesional propuesto, expectativas de formación en función de la malla curricular, nivel de satisfacción desempeño laboral formación académica, nivel de satisfacción de estrategias de aprendizaje recibidas, bibliografía utilizada actualizada, pertinencia de las práctica para fortalecer la carrera.El dato más relevante es el referido a que 109 egresados aprecian satisfacción por la formación recibida, lo cual constituye un apreciable aval para dar continuidad (y quizás perfeccionamiento debido) a los planes de estudios que se implementan. Este resultado se complementa con que 74 egresados consideran que en gran parte sus expectativas le brindan satisfacción basado en la formación recibida.

También se efectuó la indagación a los señores directivos institucionales, además de un director distrital y a nueve administrativos, para auscultar el grado de pertinencia de formación como es el caso de las competencias: generales, básicas y específicas. El comportamiento de la muestra seleccionada es como sigue:

Tabla 3: encuesta a 193 egresados de la carrera

\begin{tabular}{|c|c|c|c|c|c|c|c|}
\hline 은 & Preguntas & $\bar{\omega}$ & $\begin{array}{l}\stackrel{0}{\frac{\pi}{0}} \\
\frac{E}{0} \\
\frac{\pi}{0} \\
\end{array}$ & 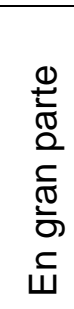 & 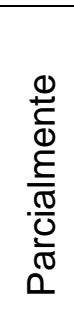 & 운 & $\begin{array}{l}\bar{\pi} \\
\stackrel{0}{0}\end{array}$ \\
\hline 1 & $\begin{array}{ll}\text { Nivel de } \\
\text { satisfacción de } \\
\text { la formación } \\
\text { recibida } \\
\end{array}$ & 109 & & 74 & & 10 & 193 \\
\hline 2 & $\begin{array}{l}\text { Cumplimiento } \\
\text { del perfil } \\
\text { profesional } \\
\text { propuesto }\end{array}$ & & 41 & 114 & 29 & 9 & 193 \\
\hline 3 & $\begin{array}{l}\text { Expectativas de } \\
\text { formación en } \\
\text { función de la } \\
\text { malla curricular }\end{array}$ & & 33 & 111 & 38 & 11 & 193 \\
\hline 4 & $\begin{array}{l}\text { Nivel } \\
\text { satisfacción } \\
\text { desempeño } \\
\text { laboral } \\
\text { formación } \\
\text { académica }\end{array}$ & & 68 & 88 & 33 & 4 & 193 \\
\hline 5 & $\begin{array}{ll}\text { Nivel } & \text { de } \\
\text { satisfacción } & \text { de } \\
\text { estrategias } & \text { de } \\
\text { aprendizaje } & \\
\text { recibidas } & \\
\end{array}$ & & 45 & 101 & 39 & 8 & 193 \\
\hline 6 & $\begin{array}{l}\text { Bibliografía } \\
\text { utilizada } \\
\text { actualizada, } \\
\text { pertinencia de } \\
\text { las práctica } \\
\text { para fortalecer } \\
\text { la carrera }\end{array}$ & & 46 & 90 & 31 & 26 & 193 \\
\hline
\end{tabular}

Tabla 4: Total de directivos institucionales encuestados.

\begin{tabular}{|c|c|c|c|c|}
\hline \multirow{2}{*}{$\sum_{\text {Wै }}^{\infty}$} & Pública & Privada & Fiscomisional & Municipal \\
\hline & 110 & 11 & 0 & 0 \\
\hline \multirow{2}{*}{$\begin{array}{l}\text { 옹 } \\
\text { ত্ }\end{array}$} & $\begin{array}{l}\text { Director } \\
\text { distrital }\end{array}$ & $\begin{array}{l}\begin{array}{l}\text { Talento } \\
\text { humano }\end{array} \\
\end{array}$ & $\begin{array}{l}\text { Rector/ } \\
\text { director }\end{array}$ & $\begin{array}{l}\text { Vicerrector } \\
\text { /subdir. }\end{array}$ \\
\hline & 1 & 9 & 101 & 10 \\
\hline 0.8 & $\begin{array}{l}\text { Servicios } \\
\text { educativos }\end{array}$ & Producción & Comercio & Distribución \\
\hline
\end{tabular}


120

0

0

Puede observarse con claridad la preponderancia de los directivos de la actividad económica y el cargo de director/vicerrector, como los que fueron estudiados en mayor medida. Los resultados alcanzados evidencian el alto nivel de satisfacción por el trabajo realizado, manifestado en que más del $85 \%$ de todos los estudiados aprueban el plan de estudios y sus programas, así como la formación que se brinda en la universidad.

Una cifra sumamente elevada que supera el $90 \%$ de los directivos estudiados considera que los servicios educativos brindados son pertinentes, pues garantiza la formación de competencias generales, básicas y específicas que permiten a los egresados realizar un buen trabajo en su práctica profesional.

\section{Tabla 5: Tendencias del mercado ocupacional}

\begin{tabular}{|c|c|c|}
\hline \multicolumn{3}{|c|}{ Perspectivas en el campo ocupacional } \\
\hline Criterios & Cantidad & Porcentaje \\
\hline $\begin{array}{l}\text { Ampliación del } \\
\text { campo ocupacional }\end{array}$ & 89 & 73,55 \\
\hline $\begin{array}{l}\text { Igualdad del campo } \\
\text { ocupacional }\end{array}$ & 9 & 7,43 \\
\hline $\begin{array}{l}\text { Reducción del } \\
\text { campo ocupacional }\end{array}$ & 23 & 19 \\
\hline & 121 & 100,0 \\
\hline \multicolumn{3}{|c|}{ Oportunidades en el campo laboral } \\
\hline Criterios & Cantidad & Porcentaje \\
\hline $\begin{array}{l}\text { Mayor oportunidad } \\
\text { de empleo }\end{array}$ & 78 & 64,46 \\
\hline $\begin{array}{l}\text { Igual oportunidad de } \\
\text { empleo }\end{array}$ & 16 & 13,22 \\
\hline $\begin{array}{l}\text { Menor oportunidad } \\
\text { de empleo }\end{array}$ & 27 & 22.31 \\
\hline & 121 & 100,0 \\
\hline \multicolumn{3}{|c|}{ Requerimientos en proceso formativo } \\
\hline Criterios & Cantidad & Porcentaje \\
\hline $\begin{array}{ll}\text { Hacer } & \text { cambios } \\
\text { drásticos } & \end{array}$ & 55 & 45,45 \\
\hline $\begin{array}{l}\text { Hacer cambios } \\
\text { moderados }\end{array}$ & 65 & 53.71 \\
\hline No hacer cambios & 1 & 0,82 \\
\hline & 121 & 100,0 \\
\hline \multicolumn{3}{|c|}{ Requerimientos de uso de TICS } \\
\hline Criterios & Cantidad & Porcentaje \\
\hline Alto uso & 102 & 84.29 \\
\hline Moderado & 19 & 15,70 \\
\hline Poco uso & 0 & 0,0 \\
\hline
\end{tabular}

Por otra parte, se hizo la indagación de las tendencias del mercado ocupacional de los maestros. Para ello se partió de la determinación de criterios profesionales, lo cual arrojó los siguientes resultados.

Es evidente que existe una percepción muy favorable hacia la consideración de que el campo laboral magisterial se ha ampliado (89 -73,55\%), que hay mayor oportunidad de empleo (78 \% $64,46)$, lo cual genera una opinión mayoritaria de que los cambios que habría que hacer en la formación magisterial sería de forma moderada (65 $53.71 \%$ ), así como que los requerimientos de uso de tics (102 - $84.29 \%$ ) tiene un alto nivel de exigencia.

En la investigación, también se tomó en cuenta la tasa de natalidad y la proyección efectuada por el Instituto de Estadísticas y Censos, (INEC), para el año 2025, datos muy relevantes para determinar los cohortes que se deben realizar en la carrera: la población infantil de 0 a 1 año de edad del 2010 correspondía a 11913 y a los niños de entre 1 y 4 años tenían un promedio de 12492 habitantes, por lo que se consideró que la tasa de nacimientos está con un decrecimiento anual de 579, de ahí que es importante este factor pues aún a pesar de ello, se tiene una proyección 9887 niños para ingresar a la educación básica a partir del año 2017.

Tabla 6: Tasa de natalidad de la provincia de El Oro, del 2010 al 2017. Fuente: Instituto Nacional de Estadísticas y Censos.

\begin{tabular}{|l|l|l|l|}
\hline 2010 & 2011 & 2012 & 21013 \\
\hline 11913 & 11334 & 10755 & 10176 \\
\hline \multicolumn{4}{|c|}{} \\
\hline 2014 & 2015 & 2016 & 2017 \\
\hline 9597 & 9019 & 8439 & 7860 \\
\hline
\end{tabular}

\section{Conclusiones}

Los estudios de la demanda de los servicios educacionales cobran cada vez mayor relevancia, por su impacto en la formación de los beneficiarios y la necesidad de elevar la eficiencia en el trabajo formativo, que además tiene una implicación en los recursos materiales y financieros que en este proceso se emplean 
De los resultados antes anotados se puede colegir la gran demanda de profesionales en el área de Ciencias de la Educación, especialidad Educación Básica, además considerando lo que se establece en los objetivos del Buen Vivir, la Ley Orgánica de Educación Superior y el Consejo de Educación Superior CES, a través del reglamento de armonización de la nomenclatura de títulos profesionales y grados académicos que confieren las instituciones de educación superior del Ecuador.

De acuerdo a los datos de campo recogidos y cotejados con las estadísticas del Ministerio de Educación, más la tasa de natalidad tomada del Instituto Nacional de estadísticas y censos, se revela un faltante de 4456 docentes especializados en Educación Básica, lo cual avala y confirma la necesidad de continuar con el plan de estudios vigente.

\section{Agradecimientos}

Se hace un público reconocimiento a los miembros del colectivo docente y dicente de la carrera de docencia en educación básica de la Universidad Técnica de Machala, así como a los directivos de los diferentes centros educativos de los niveles: inicial, básico y bachillerato de la provincia de El Oro, por su contribución al presente trabajo.

\section{Referencias}

[1] C.R. Garrocho, et al. La pertinencia social y la investigación científica en la universidad pública mexicana. Ciencia Ergo Sum, (2012), vol. 19, núm. 1, marzo-junio, pp. 24-34 Universidad Autónoma del Estado de México.

[2] Reglamento de Régimen académico, Universidad Técnica de Máchala, (2014), pp. 15.

[3] Plan Nacional del Buen Vivir del Ecuador, (2013 - 2017), (Sumak Kawsay), objetivo 4.

[4] Guía para la elaboración de los estudios de pertinencia social, factibilidad y estado del arte para la creación, modificación o liquidación de programas educativos, Consejo de Educación Superior del Ecuador, (2014).

[5] V. Cardona, Pertinencia social de los programas académicos y Guía de autoevaluación. (1995), 2da. ed. Salamanca: Asociación de Universidades Iberoamericanas de Postgrado, pp. 9.

[6] Grupo de Trabajo sobre evaluación de los postgrados en medicina social. Relatoría General. Educ Méd Salud (1992), pp, 26 - 151.

[7] Estadísticas Educativas, del Ministerio de Educación del Ecuador http://educacion.gob.ec/amie/ (2015)

[8] Instituto Nacional de estadísticas y Censos del Ecuador, www.ecuadorencifras.gob.ec (2015)

E-mail: Jubonista15@hotmail.com 